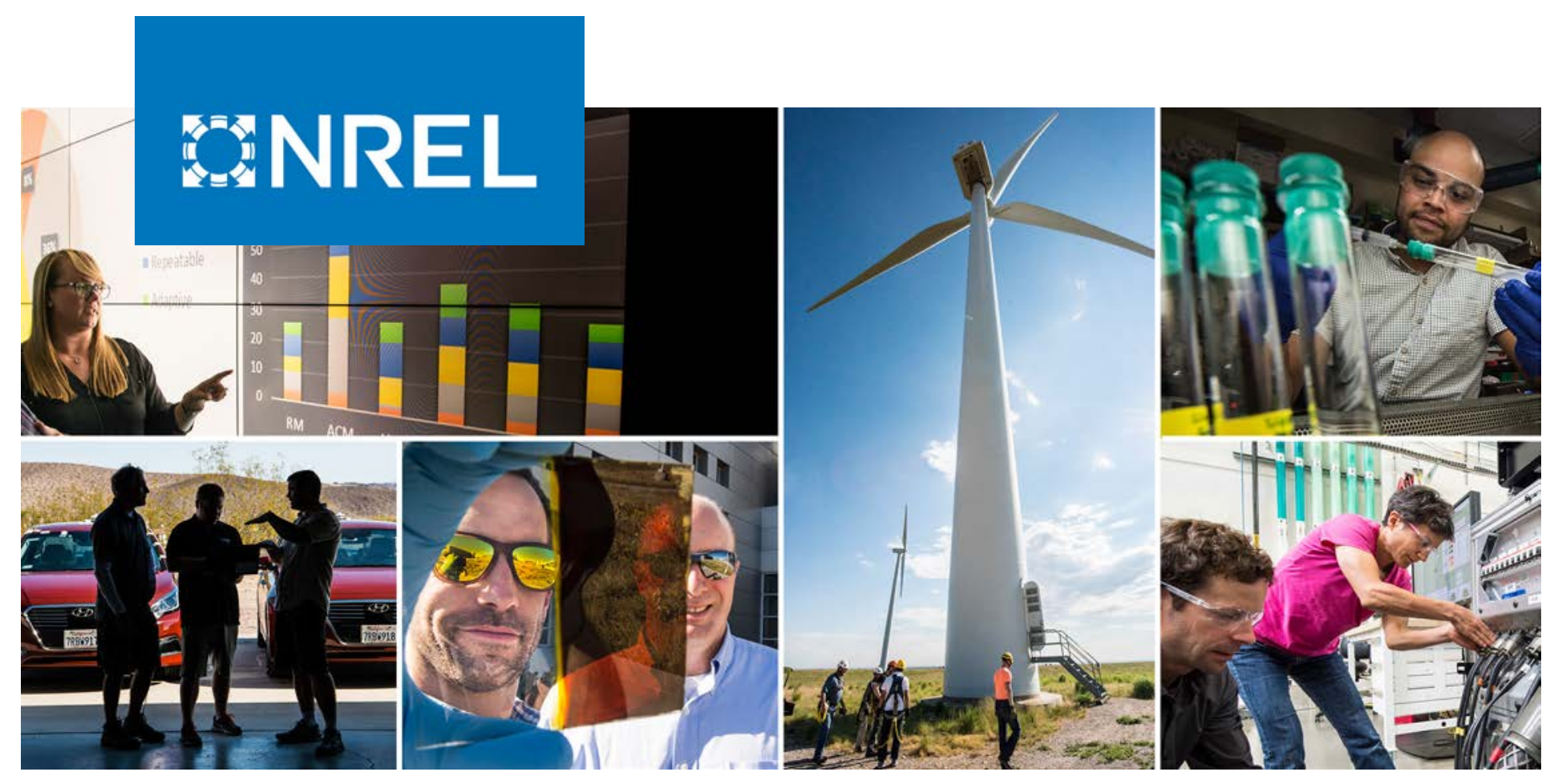

\title{
Grid Impact Analysis of Heavy-Duty Electric Vehicle Charging Stations
}

\section{Preprint}

Xiangqi Zhu, Barry Mather, and Partha Mishra

National Renewable Energy Laboratory

Presented at the 2020 IEEE Innovative Smart Grid Technologies Conference (IEEE ISGT)

Washington, D.C.

February 17-20, 2020

NREL is a national laboratory of the U.S. Department of Energy

Office of Energy Efficiency \& Renewable Energy

Operated by the Alliance for Sustainable Energy, LLC

This report is available at no cost from the National Renewable Energy Laboratory (NREL) at www.nrel.gov/publications. 


\title{
GNREL
}

\section{Grid Impact Analysis of Heavy-Duty Electric Vehicle Charging Stations}

\section{Preprint}

\author{
Xiangqi Zhu, Barry Mather, and Partha Mishra \\ National Renewable Energy Laboratory
}

\section{Suggested Citation}

Zhu, Xiangqi, Barry Mather, and Partha Mishra. 2020. Grid Impact Analysis of Heavy-Duty Electric Vehicle Charging Stations: Preprint. Golden, CO: National Renewable Energy Laboratory. NREL/CP-5D00-74838. https://www.nrel.gov/docs/fy20osti/74838.pdf.

NREL is a national laboratory of the U.S. Department of Energy Office of Energy Efficiency \& Renewable Energy Operated by the Alliance for Sustainable Energy, LLC

This report is available at no cost from the National Renewable Energy Laboratory (NREL) at www.nrel.gov/publications.

Contract No. DE-AC36-08GO28308
Conference Paper NREL/CP-5D00-74838 April 2020

National Renewable Energy Laboratory 15013 Denver West Parkway Golden, CO 80401 303-275-3000 • www.nrel.gov 


\section{NOTICE}

This work was authored by the National Renewable Energy Laboratory, operated by Alliance for Sustainable Energy, LLC, for the U.S. Department of Energy (DOE) under Contract No. DE-AC36-08GO28308. Funding provided by U.S. Department of Energy Office of Energy Efficiency and Renewable Energy Vehicle Technologies Office. The views expressed herein do not necessarily represent the views of the DOE or the U.S. Government.

This report is available at no cost from the National Renewable Energy Laboratory (NREL) at www.nrel.gov/publications.

U.S. Department of Energy (DOE) reports produced after 1991 and a growing number of pre-1991 documents are available free via www.OSTI.gov.

Cover Photos by Dennis Schroeder: (clockwise, left to right) NREL 51934, NREL 45897, NREL 42160, NREL 45891, NREL 48097, NREL 46526.

NREL prints on paper that contains recycled content. 


\section{Grid Impact Analysis of Heavy-Duty Electric Vehicle Charging Stations}

\author{
Xiangqi Zhu and Barry Mather \\ Power Systems Engineering Center \\ National Renewable Energy Laboratory \\ Golden, CO, USA \\ xiangqi.zhu@nrel.gov, barry.mather@nrel.gov
}

\author{
Partha Mishra \\ Transportation \& Hydrogen Systems Center \\ National Renewable Energy Laboratory \\ Golden, CO, USA \\ partha.mishra@nrel.gov
}

\begin{abstract}
This paper presents a grid impact analysis of heavyduty electric vehicle (EV) charging stations. In this work, heavyduty EVs have battery capacities high enough to provide a range of 250-500 miles on a single charge, such as long-haul trucks. Heavy-duty EVs will require extremely fast charging rates to reduce charging time and will induce very high charging loads (at the multiple-megawatt scale) if several vehicles charge at the same time. Therefore, analysis is needed to understand the impact of charging station loads on the electric power grid and set the baseline for developing mitigation plans and necessary system upgrades. We develop a systematic procedure to analyze the potential impact of the placement of charging stations on the grid. Charging load is modeled using a DC fast-charging station model. A voltage load sensitivity matrix approach is leveraged to investigate the challenges of placing charging stations on the feeder. Given the charging load profiles and suggested charging station locations, time-series simulations are performed on various connection points on the feeder to understand the impact. The analysis is performed on both the IEEE 34-bus system and a realistic feeder from California. Initial mitigation solutions are developed based on insights from this analysis.
\end{abstract}

Keywords-heavy-duty EV; charging station; grid impact; distribution feeder

\section{INTRODUCTION}

Electric vehicles (EVs) are increasing in quantity, and more types of EVs are entering the market. In the near future, EVs are expected to become a sizable share of the overall transportation fleet. Companies, including Tesla, are pushing heavy-duty EVs such as trucks into the market. Unlike light-duty EVs, electric trucks require faster charging rates and demand larger charge loads to enable long-distance travel. Therefore, the charging stations for heavy-duty EVs might have spiky load profiles with large peak loads ( $>1 \mathrm{MW}$ for a single port) and very high ramp rates. Hence, it is important to understand the impact of connecting heavy-duty EV charging stations to the grid.

Existing work in the literature mostly focuses on grid analysis and grid integration of light-duty EVs, with an emphasis on understanding the load behavior of the EVs and coordinating them to better aid grid operation [1]-[4]. Few studies have been conducted on investigating the impact on the grid from heavy-duty EVs and their charging stations. Lightduty EV charging loads are small loads distributed along the feeder, and the charging load profiles have small spikes, i.e., usually less than $10 \mathrm{~kW}$. In contrast, heavy-duty EV charging loads have large loads along the feeder, with load profile spikes greater than $1 \mathrm{MW}$. Because of the differences in load characteristics and charging behaviors of heavy-duty and lightduty EVs, existing analyses are insufficient to understand the grid impacts from heavy-duty EV charging stations. Therefore, we develop a systematic method to analyze and understand the grid impacts of heavy-duty EV charging stations.

The proposed methodology has three major steps: 1) charging station location investigation, 2) heavy-duty EV charging load modeling, and 3) grid impact analysis. We leverage the voltage load sensitivity matrix (VLSM) [5]-[6] to develop the voltage impact matrix (VIM) to identify the better/worse connection points for charging stations on the grid. The charging loads are modeled by a DC fast-charging charging station model [7]. Various charging load profiles are connected to different locations in the system, and the grid impact on different locations is demonstrated on both IEEE 34-bus test system and a realistic feeder from California. An initial voltage impact mitigation plan is proposed and tested on the California feeder model.

The rest of this paper is organized as follows: Section II introduces the procedure for the analysis methodology. Section III discusses the case studies performed on two distribution systems, including the IEEE 34-node test system and a realistic California feeder. Section IV concludes the paper and presents the future work plan.

\section{ANALYSIS METHODOLOGY}

This section first introduces the overall analysis approach, then further presents the methods used for each major step of the grid analysis.

\section{A. Approach Overview}

As shown in Fig. 1, the analysis methodology comprises three parts: 1) charging station location investigation, 2) charging load modeling, and 3) grid impact analysis. First, the VIM is used to partition the feeder into different zones ranging from the best locations to the worst locations. Then, the charging load is modeled considering various inputs, such as EV arriving time and EV charging rates. Finally, several representative charger connection points on the distribution system are 
selected, and the modeled charging profiles are applied to the connection points for impact analysis. The detailed approach in each step is introduced in Part B-D in this section.

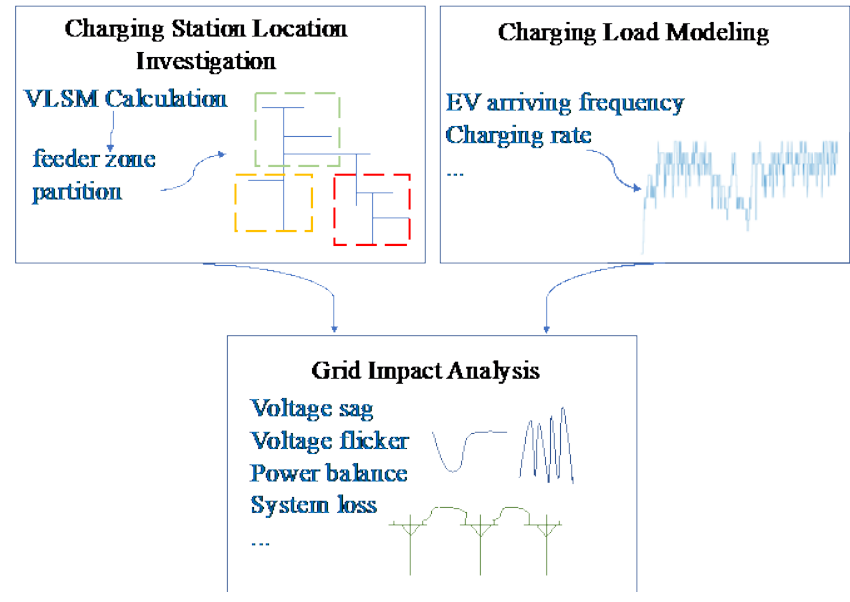

Fig. 1 Analysis approach overview

\section{B. Charging Station Location Investigation}

To identify better connection points for charging stations, VLSM is leveraged to evaluate and quantify the voltage impact brought by each connection point in the system. Two VLSMs are calculated: for real power and reactive power. As shown in (1)-(3), the voltage change, $\delta V_{i}$, at node $i$ can be calculated by multiplying the real/reactive power change, $\delta P / \delta Q$, at all the 1- $n$ nodes with the $V L S M_{P} / V L S M_{Q}$. Inside $V L S M, p_{i j} / q_{i j}$ represents the real/reactive power sensitivity factor representing the change in voltage at bus $j$ for a unit deviation of real/reactive power at bus $i$.

$|\delta V|=\left|V L S M_{P}\right||\delta P|+\left|V L S M_{Q}\right||\delta Q|$

i.e.,

$\left|\begin{array}{c}\delta V(1) \\ \vdots \\ \delta V(n)\end{array}\right|=\left|\begin{array}{ccc}p_{11} & \ldots & p_{1 n} \\ \vdots & \ddots & \vdots \\ p_{n 1} & \ldots & p_{n n}\end{array}\right|\left|\begin{array}{c}\delta P(1) \\ \vdots \\ \delta P(n)\end{array}\right|+\left|\begin{array}{ccc}q_{11} & \ldots & q_{1 n} \\ \vdots & \ddots & \vdots \\ q_{n 1} & \ldots & q_{n n}\end{array}\right|\left|\begin{array}{c}\delta Q(1) \\ \vdots \\ \delta Q(n)\end{array}\right|$

Derived from (2):

$\delta V(i)=\sum_{j=1}^{n} p_{i j} \delta P(j)+\sum_{j=1}^{n} q_{i j} \delta Q(j)$

The detailed calculation process of the VLSM can be found in our previous work [5]-[6]. Because of space constraints, the calculation process is not elaborated here.

Based on the VLSM, the VIM is developed to represent the voltage impact of a node with respect to the other nodes in the system. As shown in (4)-(5), every node has a voltage impact factor, $f_{p i} / f_{q i}$ (Calculated in (6)-(7)), representing the total voltage changes in other nodes if the real/reactive power at this node changes by one unit. The voltage impact factor is calculated as the summation of the absolute values of the sensitivity factors in each column of the VLSM.

$$
\left|\mathrm{VIM}_{P}\right|_{1 \times n}=\left|f_{p 1}, f_{p 2}, \cdots f_{p n}\right|_{1 \times n}
$$

$$
\left|\mathrm{VIM}_{Q}\right|_{1 \times n}=\left|f_{q 1}, f_{q 2}, \cdots f_{q n}\right|_{1 \times n}
$$

$$
\begin{aligned}
& f_{p i}=\sum_{j=1}^{n}\left|p_{j i}\right| \\
& f_{q i}=\sum_{j=1}^{n}\left|q_{j i}\right|
\end{aligned}
$$

Fig. 2 shows how the good locations and worse locations map out in the distribution system. The sum of the voltage impact factors for real and reactive power quantify the voltage impact of a node to the system. The greater this value is, the greater the impact on system voltages if the charging station is connected to this node. Thus, nodes with lower values are mapped as better locations, and nodes with higher values are mapped as worse locations.

\section{Larger value $f_{p 1}+f_{q 1} \quad$ Worse location

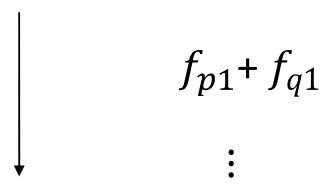 \\ smaller value $f_{p \mathrm{n}}+f_{q n} \quad$ Better location}

Fig. 2 Charging station location mapping

\section{Charging Load Modeling}

The station load profile used in this paper is generated using a DC fast-charging station model, developed to analyze the charging behaviors of EVs at a charging station [7]. A flowchart of the DC fast-charging station simulation model is shown in Fig. 3, which shows important inputs to the model, the station operational steps, and some notable outputs of the model. The model uses an agent-based modeling approach, where the vehicles and a station are defined by a set of respective properties. For a vehicle, these defining properties are its battery capacity, arrival time, initial state of charge (SOC), final desired SOC or energy demand, and a power acceptance curve. Arrival time and initial SOC are stochastic parameters whose distributions are obtained through a combination of real-world vehicle telemetry data analytics and EV system modeling [8]. Therefore, we run a Monte Carlo simulation of the station operation to obtain average performance metrics in the presence of these probabilistic parameters. The charge acceptance curve of vehicle, which is a map between SOC and maximum charging power of the battery pack and is chemistry-dependent, is used as a proxy to emulate more complex control algorithms of a battery management system (BMS). By using the charge acceptance curve, the DC fast-charging model ensures that the battery charging power is limited by either the port power capacity or by the BMS control action. A station, on the other hand, is defined by the number of charging ports, port capacity, and station capacity. When a vehicle arrives at the station, it is either plugged into a charging port if any port is empty or 
queued if all the ports are occupied. Charging is completed when the battery pack SOC reaches a predefined maximum value or a requested level of energy is added to the pack. In each Monte Carlo iteration, we simulate the station operation during a period of 1 month with time steps of 1 minute. Once the Monte Carlo iterations are complete, the DC fast-charging station software outputs a number of station performance metrics and data, including but not limited to average vehicle charging and waiting times, time-series loads of every port and the station as a whole, and battery heat generation data during charging. Example station load profiles for stations with 1, 5, and 10 installed ports and a traffic of 30 vehicles per day are shown in Fig. 4. Each port has a capacity of 1.2 MW, and the station capacity is assumed to be $1.2 \mathrm{MW}$ times the number of ports at the station. The similarity between the station loads for 5 and 10 ports in terms of the magnitude of their peaks indicates that for 30 vehicles per day, the charging demand can be met by 5 installed ports or fewer.

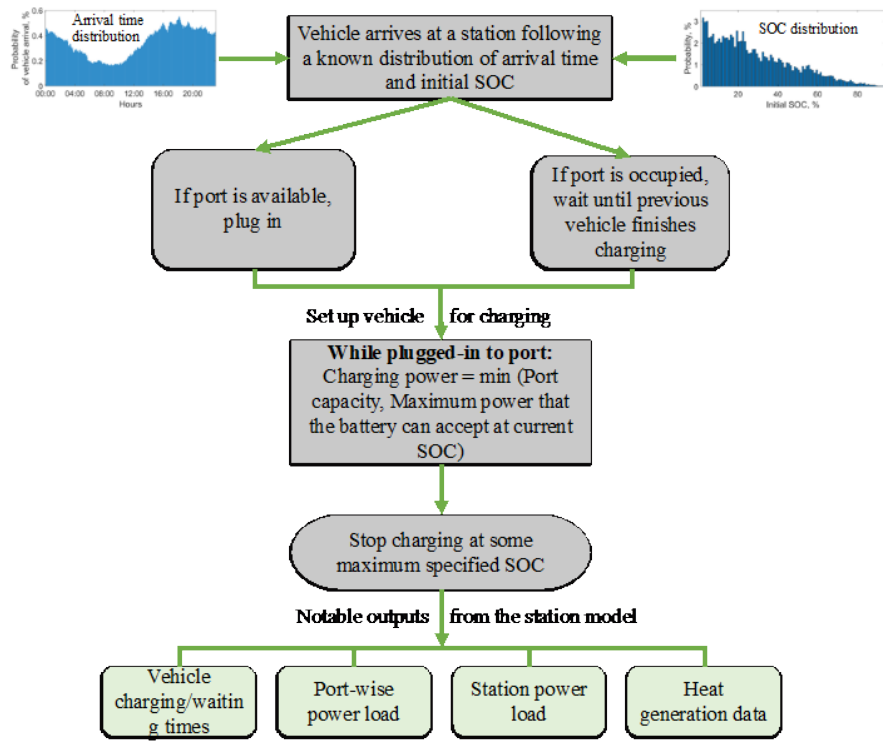

Fig. 3 Flowchart of the operation of a DC fast-charging station model

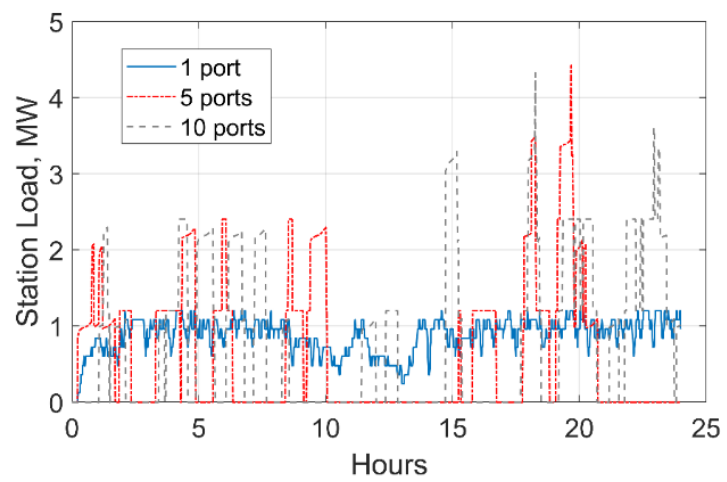

Fig. 4 Station load profiles for stations with different numbers of charging ports

\section{Grid Impact Analysis and Mitigation}

After the better/worse locations have been mapped out on the distribution system, various modeled charging load profiles are connected to different connection points on these mapped locations throughout the system. A time-series analysis is performed to analyze the impact on the system for different placements of these heavy-duty EV charging stations. Voltage violations and ramps are evaluated in our initial grid impact analysis.

The initial voltage impact mitigation approach using reactive power support functions of the smart chargers is proposed and validated in this paper. A comprehensive impact mitigation plan will be introduced in detail in a following paper.

\section{CASE STUDY}

This section discusses the analysis performed on two distribution system models: the IEEE 34-node test system and a realistic California distribution feeder model. Voltage violations and voltage ramps are analyzed for both the cases. An initial mitigation plan is tested on the realistic California feeder model. The time-series simulation resolution is 1 minute.

\section{A. Sample Charging Load Profiles}

In the base case, where no charging stations are placed on the feeder, the load profiles at each node are modeled using the method developed in our previous work [9]-[11]. The load profiles along the feeder are diversified with various patterns and different variations. Fig. 5 shows three load profile samples.

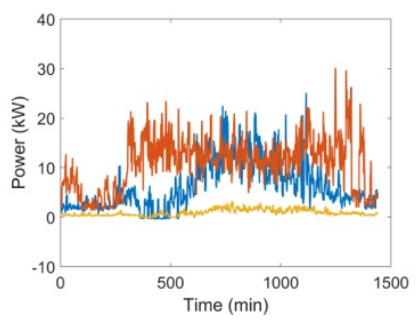

Fig. 5. Sample load profiles

The charging load profiles are modeled with the approach discussed in Part C of Section II. Fig. 6 shows the two charging load profiles used for grid impact analysis in this paper. Fig. 6(a) shows the total charging profiles of five charging ports, which is applied to the analysis of the IEEE 34-node test system. Fig. 6(b) shows the charging profile used for simulation of the realistic feeder, which represents the load profile of one charging port.

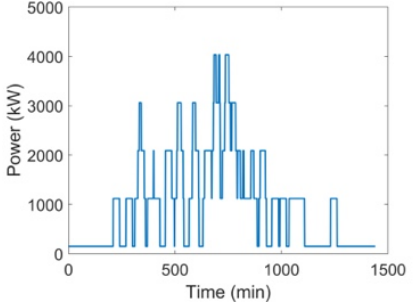

(a)

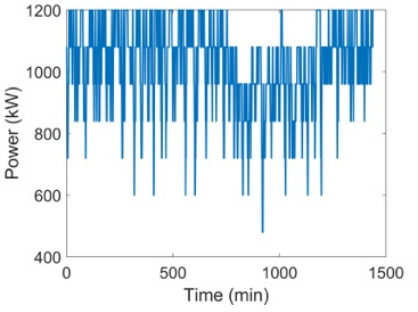

(b)
Fig. 6. Sample charging load profiles 


\section{B. IEEE 34-Bus System}

Based on the VIM, the nodes at the IEEE 34-node test feeder are sorted out and clustered into different groups. As shown in Fig. 7, sample nodes in each group are shown in different colored zones. Green, yellow, and red zones represent good, mediocre, and worst locations, respectively.

Four case studies are performed, with the base case representing the case without any charging stations; and Case 1, Case 2, and Case 3 representing good, mediocre, and worst locations, respectively. Five nodes inside each level of location are selected to place the charging stations. Therefore, for each case, five different charging loads are connected to the five selected locations in each group. The summation of the five loads is the profile shown in Fig. 6(a), with a peak charging load of approximately $4 \mathrm{MW}$.

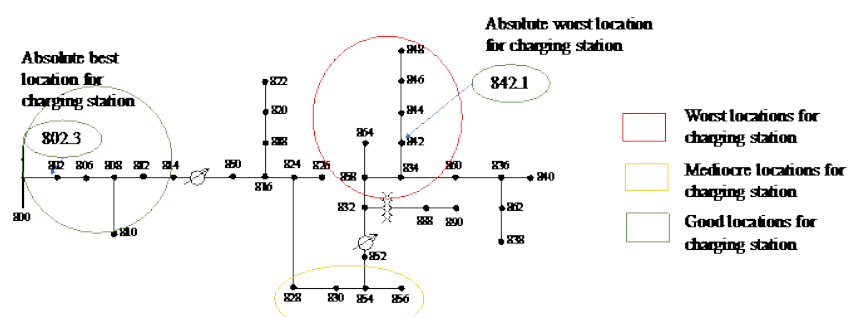

Fig. 7. Partition example for IEEE 123-bus feeder

Representative time-series voltage results in the three cases are plotted in Fig. 8, with (a)-(c) representing good, mediocre, and worst location, respectively. The location on the feeder is also shown in each caption, where 816,840 , and 844 represent bus numbers; and $.2, .3$, and .2 represent phases $\mathrm{B}, \mathrm{C}$, and $\mathrm{B}$, respectively. Comparing the three figures shows that the voltage profile is not greatly affected when the charging stations are placed at the good locations; however, connecting charging stations to other locations, especially worst locations, causes very large voltage sags and induces a lot of voltage violations.

Fig. 9 shows the statistical analysis of the 1-day time-series voltage results. Figs. 9 (a)-(c) show the distributions of the minimum/maximum daily voltage of all the nodes in the system, for all the cases, including the base case and cases 1-3. From Case 1 to Case 3, the number of voltage violations (greater than 1.05 and less than 0.95 ) increases, which shows that case 1 (good locations) have the best capability to accommodate heavy-duty charging stations. The voltage ramp distribution shown in Fig. 9(d) shows that Case 1 has smaller voltage ramps, whereas cases 2 and 3 have larger voltage ramps.

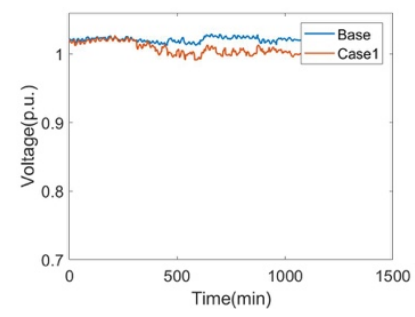

(a) Good location (816.2)

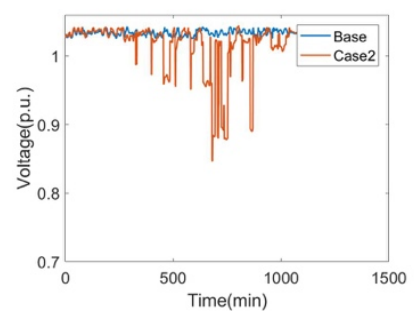

(b) Mediocre location (860.3)

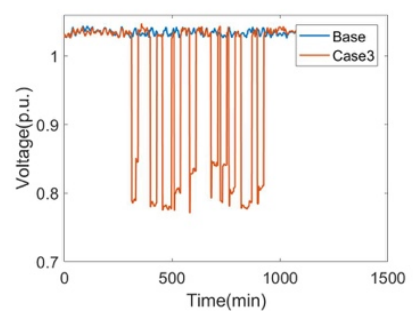

(c) Worst location (844.2)

Fig. 8 Voltage profile after charging station connected

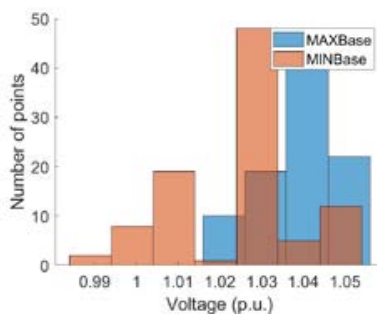

(a) Base case

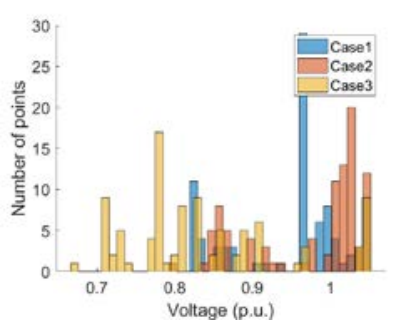

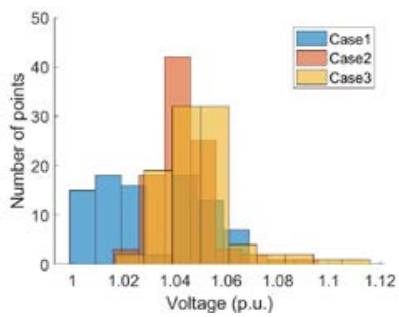

(b) Daily maximum voltage distribution

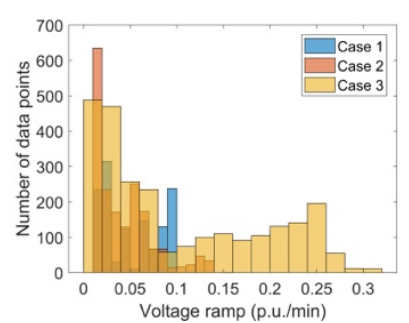

(d) Voltage ramp distribution
Fig. 9 Statistical analysis of voltage results

\section{California Realistic Feeder}

Using the method discussed in Part B of Section II, the nodes in this California feeder are sorted, and each node is marked as a good, mediocre, or worst connection point. In this realistic feeder case, one charging port is connected to one node at a time. As shown in Fig. 10, three locations are selected along the feeder.

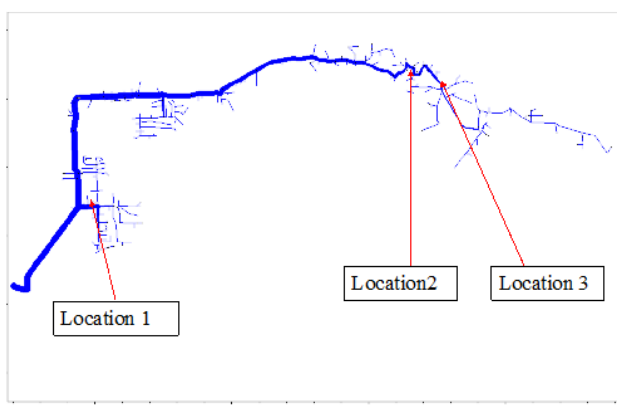

Fig. 10 Charging station placement on California feeder

The time-series voltage profiles for the three cases are shown in Fig. 11. Location 1 has better capability to incorporate the charging station than locations 2 and 3; however, even Location 1 cannot handle the heavy and spiky charging loads: the voltages decrease to less than 0.95 p.u. 
An initial mitigation plan is tested on this feeder to help the feeder better accommodate a charging station. In this mitigation case, smart chargers provide reactive power support while pulling real power heavily from the grid. Fig. 12 shows the testing results of two mitigation cases. Fig. 12(a) shows the case where a smart charger provides reactive power support, which equals $60 \%$ of the power amount it pulls from the grid; whereas Fig. 12(b) shows a 100\% support case. The $100 \%$ support case is able to maintain the voltage within the [0.96 1.05] limit, whereas the $60 \%$ support case is still struggling around the minimum limitation edge.

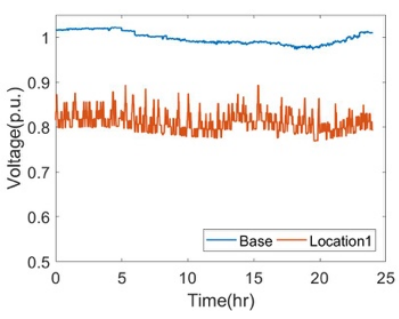

(a) Location 1

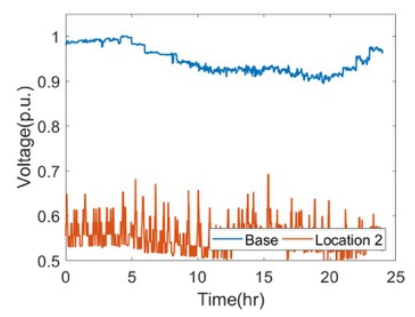

(b) Location 2

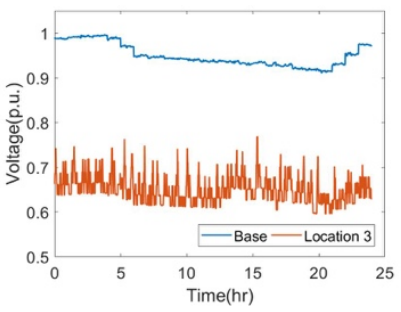

(c) Location 3

Fig. 11. Voltage profiles for three charging station locations

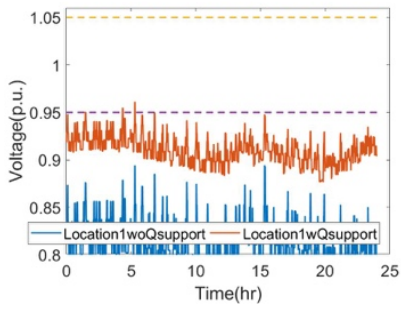

(a) $60 \% \mathrm{Q}$ support

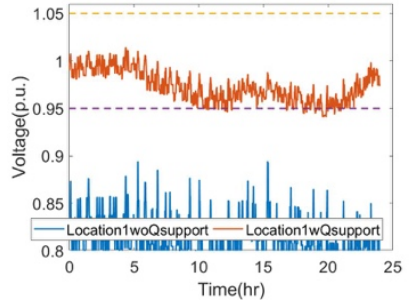

(b) $100 \%$ Q support
Fig. 12. Q support for voltage sag mitigation

\section{CONCLUSION}

This paper presented a systematic methodology for grid impact analysis of heavy-duty EV charging stations. Three major steps were introduced: investigation of charging station location, charging load modeling, and grid impact analysis. The proposed method was tested on two distribution systems: the IEEE 34-bus system and a California realistic feeder model. The results demonstrated the voltage impact that the charging station might bring to the system. The proposed analysis approach is not system-dependent and can be generalized to other feeders. Based on the analysis results, an initial mitigation plan using smart charger was proposed and tested. A comprehensive impact mitigation plan using both a smart charger and on-site distributed energy resources will be developed in the future.

\section{ACKNOWLEDGMENT}

This work was authored by the National Renewable Energy Laboratory, operated by Alliance for Sustainable Energy, LLC, for the U.S. Department of Energy (DOE) under Contract No. DE-AC36-08GO28308. Funding provided by U.S. Department of Energy Office of Energy Efficiency and Renewable Energy Vehicle Technologies Office via the 1+ MW Medium Duty/Heavy Duty Vehicle Project. The views expressed in the article do not necessarily represent the views of the DOE or the U.S. Government. The U.S. Government retains and the publisher, by accepting the article for publication, acknowledges that the U.S. Government retains a nonexclusive, paid-up, irrevocable, worldwide license to publish or reproduce the published form of this work, or allow others to do so, for U.S. Government purposes.

\section{REFERENCES}

[1] Clement-Nyns, K., Haesen, E., \& Driesen, J. (2009). The impact of charging plug-in hybrid electric vehicles on a residential distribution grid. IEEE Transactions on power systems, 25(1), 371-380.

[2] Richardson, D. B. (2013). Electric vehicles and the electric grid: A review of modeling approaches, Impacts, and renewable energy integration. Renewable and Sustainable Energy Reviews, 19, 247-254.

[3] Putrus, G. A., Suwanapingkarl, P., Johnston, D., Bentley, E. C., \& Narayana, M. (2009, September). Impact of electric vehicles on power distribution networks. In 2009 IEEE Vehicle Power and Propulsion Conference (pp. 827-831). IEEE.

[4] Lopes, J. A. P., Soares, F. J., \& Almeida, P. M. R. (2010). Integration of electric vehicles in the electric power system. Proceedings of the IEEE, 99(1), 168-183.

[5] Zhu, X., Wang, J., Mulcahy, D., Lubkeman, D. L., Lu, N., Samaan, N., \& Huang, R. (2017, July). Voltage-load sensitivity matrix based demand response for voltage control in high solar penetration distribution feeders. In 2017 IEEE Power \& Energy Society General Meeting (pp. 1-5). IEEE.

[6] Zhu, X., Wang, J., Lu, N., Samaan, N., Huang, R., \& Ke, X. (2018). A hierarchical vlsm-based demand response strategy for coordinative voltage control between transmission and distribution systems. IEEE Transactions on Smart Grid.

[7] E. Y. Ucer, M. C. Kisacikoglu, F. Erden, A. Meintz and C. Rames, "Development of a DC fast charging station model for use with EV infrastructure projection tool," 2018 IEEE Transportation Electrification Conference and Expo (ITEC), Long Beach, CA, pp. 904-909, 2018.

[8] P. P. Mishra, E. Miller, S. Gupta, S. Santhanagopalan, K. Bennion, A. Meintz, and K. Walkowicz, "A framework to analyze the requirements of a multiport megawatt-level charging station for heavy-duty electric vehicles," under review.

[9] M. Chamana, B. Mather. "Variability extraction and synthesis via multiresolution analysis using distribution transformer high-speed power data." IEEE Intelligent System Application to Power Systems (ISAP), 2017

[10] Zhu, X., \& Mather, B. (2018, August). DWT-Based Aggregated Load Modeling and Evaluation for Quasi-Static Time-Series Simulation on Distribution Feeders. In 2018 IEEE Power \& Energy Society General Meeting (PESGM) (pp. 1-5). IEEE.

[11] Zhu, X., \& Mather, B. (2019, August). Data-Driven Load Diversity and Variability Modeling for Quasi-Static Time-Series Simulation on Distribution Feeders . In 2019 IEEE Power \& Energy Society General Meeting (PESGM). IEEE. 